\title{
Revising Algorithm for Pathology Image Stitching
}

\author{
Haishun Wang ${ }^{\mathrm{a}}$, Limin Chen ${ }^{\mathrm{b}}$ \\ ${ }^{a}$ College of Information \& Technology, Zhejiang Chinese Medical University, Hangzhou, Zhejiang. P.R.China \\ ${ }^{b}$ Shanxi University, Taiyuan,Shanxi, P.R.China
}

\begin{abstract}
Image stitching for pathological slice splices several adjacent images into an integrated seamless picture which is significant in remote medicine, especially remote diagnosis. However, because of limitations of image acquisition method, some mismatch could occur. This paper proposed a new image mosaic revising algorithms based on the relativity of adjacent images. After experimental verification, the 20 groups inaccurate pathological mosaic images were revised rapidly and accurately with error controlled within a pixel. It is proved that the algorithm is effective in revising the error matching in pathological images mosaic.
\end{abstract}

Index Terms: image mosaic; pathological slice image stitching; remote diagnosis; image mosaic error revising

(C) 2012 Published by MECS Publisher. Selection and/or peer review under responsibility of the Research Association of Modern Education and Computer Science.

\section{Introduction}

It is difficulty to remote diagnosis by pathological slice which only observing the tiny area with high time pathologic images. By image mosaic, the several adjacent pathological slices can be stitched as an integrated seamless picture. This requires that splicing areas transit naturally without any gaps in color and structure [1]. In general, there are usually two types of seams between images, one is different brightness gap caused by uneven light exposure, the other is the geometric structure gap showing dislocation, overlapping and separation caused by image distortion or image registration error.

Literature [2] has analyzed and attributed the formation of the geometric structure gap to adjacent pixels. The image's overlapping parts make a relative small rotation during image acquisition. The subsequent variation is difficult to correct. Some scholars have pointed to the solution of such problems, for example, Jia as in [3] adopted the structured deformation approach to deal with structural dislocation, Levin as in [4] used GIST method, which is to optimize the gradient strength in the overlapping region to achieve the image mosaic. However, these methods are of limited use in solving structure dislocation issues.

When using the 2D image acquisition scan mode, frame to frame method will cause accumulation of the adjacent image alignment error, resulting in mismatching. At present, some global optimization methods are 
used by the image splicing while adjusting all alignment parameters, it can effectively eliminate the error accumulation in the image mosaic [4-6], but it is rather slow and unable to effectively deal with large scale image mosaics. Literature [7] has proposed an improved method with certain effects, however the proposed method is still not ideal for applications requiring higher accuracy.

Literature [8] proposed the basic principle for images mosaic revising, that is while the four adjacent images were spliced rightly, there is the relevance of their position, when the three images can be correctly spliced, the fourth correctly spliced between images is determined, so that one pair images' mismatch can be revised.

This paper proposes a modified algorithm, which is based on the relevance of the position between adjacent image mosaic. In order to highlight the core method of image stitching, while not losing sight of practical application, this paper focuses on pathological microscope image stitching, in which those data changes by relative small rotations or focusing the target twice after moving are simply attributed to splicing error processing.

\section{Funds of the revising algorithm}

The image mosaic can be processed from the horizontal and vertical direction. As long as two images are stitched successfully, the uniform area should be overlapped. Fig.1 is a horizontal stitching case, and the horizontal splicing column of graph A is named pjh, which is the projection of the initial vertical edge of graph B. The jcv is called vertical interleaving distance, which is the vertical diverge distance of the overlapped area through horizontal stitching. Similarly, vertical stitching, the pjv is the row of vertical splicing and jch is the horizontal staggered distance.

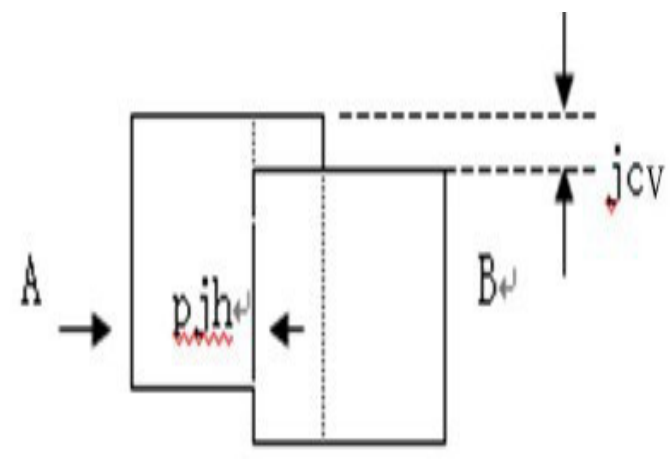

Fig. 1. Horizontal splicing

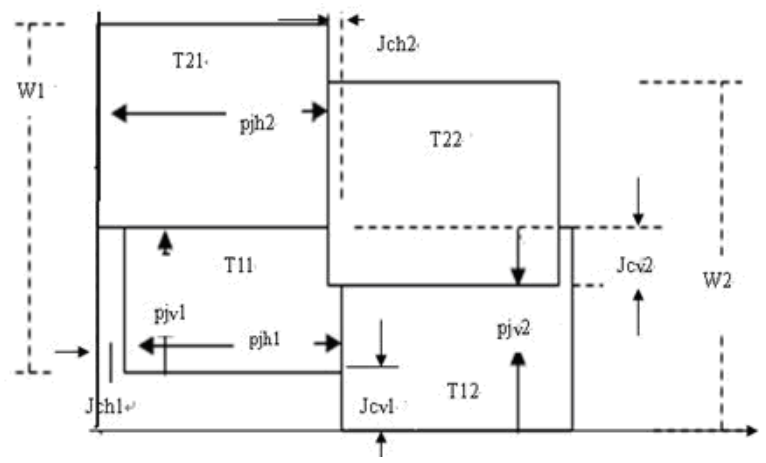

Fig. 2. Four neighbor image splicing 
When considering the four adjacent images splicing with two rows and two columns, there are two horizontal splicing, and two vertical stitching. Shown in Fig. 2, T11, T12 and T21, T22 are the first row and second row of the horizontal adjacent images, set pjh1, jcv1 and pjh2, jcv2 are their horizontal splicing columns and vertical interleaving distance, T11, T21 and T12, T22 respectively, for the first and second columns vertically adjacent image, pjv1, jch1 and pjv2, jch2 are two vertical splicing rows respectively and two horizontal staggered distance. Above the four adjacent images form an independent splicing unit, according to their relative of the location, there are following equations.

$$
\begin{aligned}
& \text { pjh1 }=\text { pjh2-jch1 }+ \text { jch2 } \\
& \text { pjv1= pjv2-jcv1+jcv2 }
\end{aligned}
$$

Equations (1) and (2) can be proved easily, using the relationship between the positions in Fig.2.

According to the basic relation of image mosaic there are following properties.

The property 1: The horizontal stitching column and vertical stitching row determined the stitching direction and position respectively and affected the overlap situation, while the row or column will be lost by more overlap and increased by less overlap. Moreover, the horizontal and vertical interleaving situation relate to the staggered distance in the vertical stitching direction, shown as Fig.3.

The property 2: Equations (1) and (2) are independent. They represent the horizontal and vertical alignment situation. When splicing correctly, (1) and (2) are established, the converse is not true. Because each equation, if and only if the three parameters are determined, then has a unique solution.

The relationship between splicing parameter and interleaving distance should meet (1) and (2), otherwise, need to be amended. The amount of revising called parameter modified weight which can be reasoned from (1) and (2) directly.

$$
\begin{aligned}
& \mathrm{Xh}=\text { pjh2-pjh1+jch2-jch1 } \\
& \mathrm{Xv}=\text { pjv2-pjv1+jcv2-jcv1 }
\end{aligned}
$$

Where $\mathrm{Xh}$ and $\mathrm{Xv}$ stand as the weight of parameter modified in horizontal and vertical mosaic direction which reflecting the stitching state. While the weight is zero, (1) and (2) come into existence and the parameter do not need modification generally. Equations (1) and (2) and some of the above properties will become the basic theory and modified splicing basis.

\section{Processing of the revising algorithm}

The literature [8] shows that in the mosaic units as long as three sets of pictures are spliced correctly, the fourth one on the map will also be correctly spliced. Figs. 1 and 2 show that there are eight parameters of four images in graphs .when three pairs of pictures can be correctly spliced, then pjh1, jcv1, pjh2, jcv2, pjv1, jch1, a total of six parameters are known and the pjv2 and jch2 can be calculated respectively with Eqs. 1 and 2 according to the known parameters. Therefore, the above conclusion is correct. The processing is as following.

a) Processing the first row and first column. While splicing several individual images into an integrate one, positioning of the image is the key point. Mosaic unit of Fig. 2 shows that in the T11 the jch1 and jcv1 determine the horizontal and vertical position, if jcvl protrudes upward, take jcv1 as an initial value, otherwise jcvl $=0$ (taking the edge of T11 as the initial value). Therefore, the position variables of T11 should be modified as $\mathrm{y}+$ $\mathrm{jcv} 1, \mathrm{x}+\mathrm{jch} 1$. It can be deduced that the vertical staggered parameter is the location basis in the vertical 
direction of the first row of the image and horizontal one is the one in level direction. Specific treatment should be considered when variable parameters are present, further simplification is required.

By studying the regularity of level and vertical edge changes in image splicing, we summarized the following 8 simplified rules (as in Fig.2).

Vertical splicing appears when owe overlap. 1) while splicing T11 and T12, modifying the jcv1.2) while splicing T21 and T22, if jcv1>0 modifying (pjv1 or jcv1) or pjv2 else modifying pjv1 or (jcv1 or pjv2).

Vertical splicing occurs in excessive overlapping. 3). while splicing T12 and T22, if cjv1>0 modifying the pjv2, else: modifying the (pjv2,or jcv1) .4) while splicing T11 and T21, if jcv1>0 modifying the (pjv1 or jcv1>0) else modifying the pjv1.

Like the same approach for vertical stitching, other four rules to process the horizontal diverge or overlap.

After processing the first row and first column, the left and below parameters in the parameter table can be regarded as constant.

b) Processing T22 directly while splicing the mosaic unit. Known from previous sections, except for the first row and first column, the left and below parameters in the parameter table is constant so that the processing mosaic unit can be changed to splice the T22 directly. It may be related to both vertical and horizontal stitching which can be determined by Eqs. 3 and 4, if $\mathrm{Xh}=0$ there is nothing to do for pjh 2 and jch2, or else one would only need to modify pjv2 and jcv2 which is related by Eq.2 and can modify pjv2 directly, as well as for horizontal splicing, modify pjh2 straight away.

c) While the parameters in the unit which $\mathrm{Xh}=0$ and $\mathrm{Xv}=0$, modification is unnecessary.

After the above simplification, it is basically concentrated in 1 to 2 parameter changes, greatly reducing the computational complexity, making revision possible. The remaining selection work will be conducted with help from eyesight.

\section{Experiment results}

To verify the effectiveness and practicability of the revising algorithm, an experiment revised 20 group images which each group consisted of 16 images, and size of image $512 \times 512$, overlapped area about 100 pixels.

\subsection{Experiment data and result of image in group $B$}

Fig. 3 is the original result of image splicing in B group. In order to facilitate inspection and correction, the following procedures are proposed:

a) Modify the brightness of the adjacent images so as to check the gap easily.

b) In order to realize accurate splicing, this paper does not use smooth, and the yellow flat circles point out the wrong stitching places. Magnification is used.

c) In order to facilitate inspection and correction, the horizontal and vertical splicing parameters are put together respectively to create two parameter tables automatically, shown as in Table I and Table II, which are composed of each unit splicing parameters and in turn. The pjh, and pjv in the table are the mosaic columns and rows respectively, jch and jcv are the horizontal and vertical staggered parameters.

Using (1) and (2) calculated and created the level and vertical modified weight table automatically as shown in table III and table IV. According to table III and table IV, to check the stitching situation, if the value of item in the tables is not zero, usually has to modify the parameters of corresponding splicing unit. 

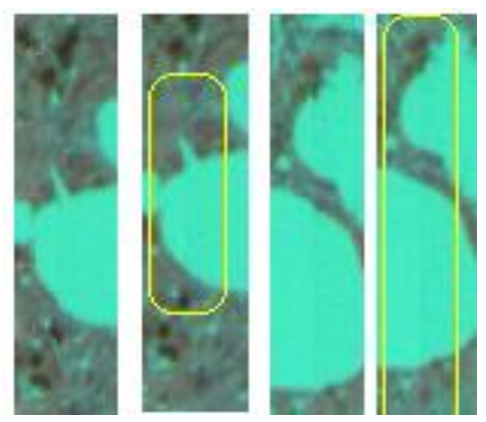

Fig. 3. Image stitched by 16 images

\subsection{Compared before and after revising}

After revised by the above method, the image integrated by 16 pictures is basically intact. A comparison before and after revising is in the Figs.4-6. The splicing mistakes have been modified perfectly.

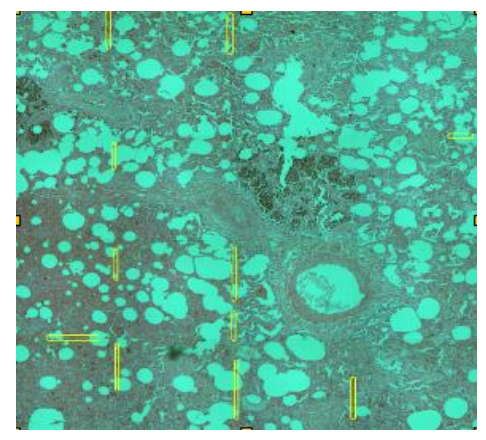

Fig. 4. Image B stitched by level

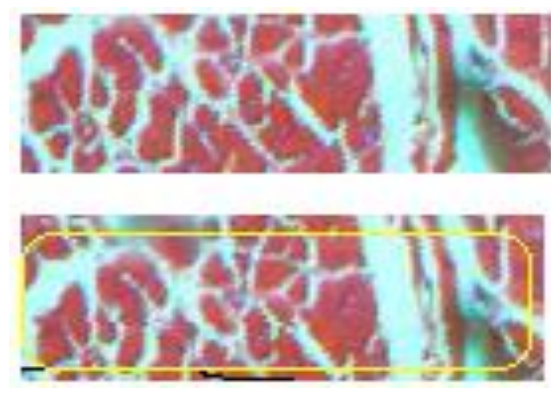

Fig. 5. Image $\mathrm{C}$ stitched by vertical 1 


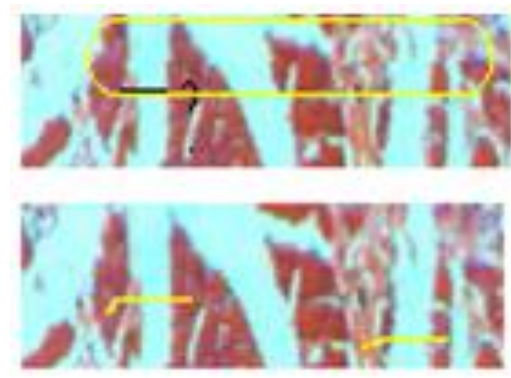

Fig. 6. Image $\mathrm{C}$ stitched by vertical 2

\subsection{Dealing with serious splicing errors in images of group $S$}

There are greater splicing errors in images of $\mathrm{S}$ group, even appearing hollow phenomenon in Fig. 7. Table $\mathrm{V}$ and table VI are revised value table. The upper left corner in the image confirmed splicing unit with significant errors. However, from the table, it can be found, that the splicing errors are small in other parts, providing the possibility to correct.

For example, Fig.8 is the result of stitching the upper left corner, in the figure there are two significant horizontal and vertical gaps, and the stitching pattern on both sides are not similar at all, indicating that the stitched row and column involve a major error. Fig. 9 is a revised image, comparing it with Fig.7, it can be found that the stitched row and column on the mosaic unit of upper left corner of the picture have undergone great changes, which reflects the strong revising capability of algorithm.

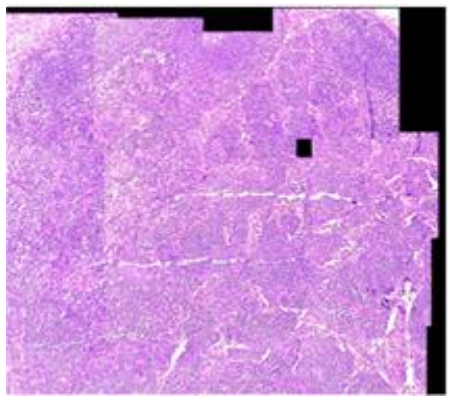

Fig. 7. Original stitched image of group $\mathrm{S}$

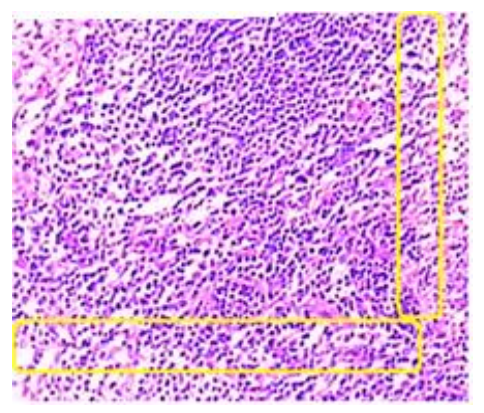

Fig. 8. Upper left corner stitched errors in group $\mathrm{S}$ 


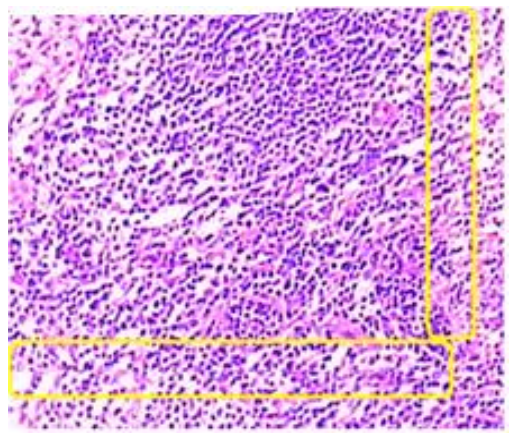

Fig. 9. Revised stitched image of group S

Table 1. level parameter table

\begin{tabular}{ccccccc}
\hline $\boldsymbol{j} \boldsymbol{c h}$ & $\boldsymbol{p j h}$ & $\boldsymbol{j} \boldsymbol{c h}$ & $\boldsymbol{p j h}$ & $\boldsymbol{j} \boldsymbol{c h}$ & $\boldsymbol{p j h}$ & $\boldsymbol{j} \boldsymbol{c h}$ \\
\hline \multirow{2}{*}{26} & 376 & 17 & 383 & & 380 & \\
& 367 & 7 & 368 & 3 & & 1 \\
1 & 374 & 1 & 370 & 9 & 376 & 8 \\
1 & 375 & & 375 & 6 & 369 & 1 \\
\hline
\end{tabular}

Table 2. vertical parameter table

\begin{tabular}{|c|c|c|c|c|c|c|}
\hline pjv & $j c v$ & pjv & $j c v$ & pjv & $j c v$ & $p j v$ \\
\hline & 1 & & 1 & & 1 & \\
\hline \multirow[t]{2}{*}{363} & & 363 & & 364 & & 364 \\
\hline & 1 & & 1 & & 1 & \\
\hline \multirow[t]{2}{*}{359} & & 359 & & 359 & & 359 \\
\hline & -1 & & 1 & & 1 & \\
\hline \multirow[t]{2}{*}{353} & & 353 & & 353 & & 354 \\
\hline & 1 & & 1 & & 1 & 0 \\
\hline
\end{tabular}

Table 3. Level modified weight

\begin{tabular}{ccc}
\hline 0 & 1 & 1 \\
\hline-1 & 0 & -1 \\
\hline-1 & 0 & 2
\end{tabular}


Table 4. Vertical modified weighT

\begin{tabular}{lll}
\hline 0 & 1 & 0 \\
\hline 2 & 0 & 0 \\
\hline-2 & 0 & 1 \\
\hline
\end{tabular}

\section{Conclusion}

Through this experiment, the pathological mosaic images of 20 groups have been revised by the splicing correction algorithm with good results. This algorithm is suitable for occasions requiring high precision and involving difficult data acquisition, and it has a strong ability to revise mosaic image with effectiveness. However, it is necessary to improve process automation for final parameters selection and to expand the scope of stitching while optimizing the system.

\section{References}

[1] Zhang Xufeng, Yan Zhuangzhi, Liu Shupeng. A Method of Image Mosaic from Microscopic Images.Shanghai Biomedical Engineering.2005, vol.26, (1), pp.13-16.(in Chinese)

[2] Wang Guo-jun, Lou Xiao, Chen Li-min. A Stitching Algorithm Based on Area Structure Character of Image for Pathologic Slice. Chinese Journal of Biomedical Engineering. 2004, vol. 23, (2), pp.121-126.(in Chinese)

[3] Jiaya Jia and Chi-Keung Tang.Image Stitching Using Structure Deformation.IEEE Transactions on Pattern Analysis and Machine Intelligence(TPAMI).2008,vol. 30, (4), pp.:617-631.

[4] Anat Levin, Assaf Zomet, Shmuel Peleg etc. Seamless Image Stitching in the Gradient Domain. Hebrew University.2003, vol. 82, pp. 377-389.

[5] Shum H Y, Szeliski R. Construction and Refinement of Panoramic Mosaics with Global and Local alignment. Proceedings of the 6th International Conference on Computer Vision,Bombay. 1998, vol. 9, pp. 53956.

[6] Sawhney H S,Kumar R.VideoBrush.Experiences with Consumer Video Mosai. Proceedings of the Workshop on Applications of Computer Vision, Princeton.1998, pp. :386-389.

[7] Miao Ligang, Yue Yongjuan, Peng Silong. Microscopic Image Mosaic Based on 2D Grid Graph. Computer Engineering.2007, vol. 33, (12), pp. 49-51.(in Chinese)

[8] Ding Ying, Hong Jiguang. Distinguishing and Solving of Pseudo Match in Image Mosaic .Journal of Image and Graphics.1999,(10), pp. 886-890.(in Chinese) 